\title{
BOUNDARY VALUE PROBLEMS FOR ELLIPTIC PSEUDO-DIFFERENTIAL OPERATORS II
}

\author{
KAZUAKI TAIRA
}

Department of Mathematics, Hiroshima University, Higashi-Hiroshima 739, Japan

Dedicated to Professor Shinzo Watanabe on his 60th birthday

\begin{abstract}
The purpose of this paper is to study boundary value problems for elliptic pseudo-differential operators which originate from the problem of existence of Markov processes in probability theory, generalizing some results of the previous work $[\mathbf{9}, \mathbf{1 0}]$. Our approach has a great advantage of intuitive interpretation of sufficient conditions for the unique solvability of boundary value problems in terms of Markovian motion. In fact, we prove that if a Markovian particle moves incessantly both by jumps and continuously in the state space, not being trapped in the set where no reflection phenomenon occurs, then our boundary value problem is uniquely solvable in the framework of Sobolev spaces of $L^{p}$ style.
\end{abstract}

\section{Introduction}

Let $\Omega$ be a bounded domain of Euclidean space $\mathbf{R}^{n}$, with $C^{\infty}$ boundary $\partial \Omega$; its closure $\bar{\Omega}=\Omega \cup \partial \Omega$ is an $n$-dimensional, compact $C^{\infty}$ manifold with boundary.

Let $W$ be a second-order, elliptic pseudo-differential operator with real coefficients such that

$$
\begin{aligned}
W u(x)= & P u(x)+S_{r} u(x) \\
:= & \left(\sum_{i, j=1}^{n} a^{i j}(x) \frac{\partial^{2} u}{\partial x_{i} \partial x_{j}}(x)+\sum_{i=1}^{n} b^{i}(x) \frac{\partial u}{\partial x_{i}}(x)+c(x) u(x)\right) \\
& +\int_{\Omega} s(x, y)\left[u(y)-\sigma(x, y)\left(u(x)+\sum_{j=1}^{n}\left(y^{j}-x^{j}\right) \frac{\partial u}{\partial x_{j}}(x)\right)\right] d y .
\end{aligned}
$$

Here:

(1) $a^{i j} \in C^{\infty}\left(\mathbf{R}^{n}\right), a^{i j}=a^{j i}$ and there exists a constant $a_{0}>0$ such that

$$
\sum_{i, j=1}^{n} a^{i j}(x) \xi_{i} \xi_{j} \geq a_{0}|\xi|^{2}, \quad x \in \mathbf{R}^{n}, \xi \in \mathbf{R}^{n}
$$

1991 Mathematics Subject Classification. Primary 35S15, 35J25; Secondary 60J60, 60J50.

Key words and phrases. Boundary value problems, pseudo-differential operators, Markovian motion. 
(2) $b^{i} \in C^{\infty}\left(\mathbf{R}^{n}\right)$.

(3) $c \in C^{\infty}\left(\mathbf{R}^{n}\right)$ and $c \leq 0$ in $\Omega$.

(4) The integral kernel $s(x, y)$ is the distribution kernel of a properly supported, pseudo-differential operator $S \in L_{1,0}^{2-\kappa}\left(\mathbf{R}^{n}\right), \kappa>0$, which has the transmission property with respect to $\partial \Omega$, and $s(x, y) \geq 0$ off the diagonal $\left\{(x, x): x \in \mathbf{R}^{n}\right\}$ in $\mathbf{R}^{n} \times \mathbf{R}^{n}$. Here we recall (see $[4,6,7]$ ) that $S$ is said to have the transmission property with respect to $\partial \Omega$ if the restriction of $S\left(u^{0}\right)$ to $\Omega$ has a $C^{\infty}$ extension to $\mathbf{R}^{n}$ for every $u \in C^{\infty}(\bar{\Omega})$, where $u^{0}$ is the extension of $u$ to $\mathbf{R}^{n}$ by zero outside $\bar{\Omega}$.

(5) The function $\sigma(x, y)$ is a $C^{\infty}$ function on $\bar{\Omega} \times \bar{\Omega}$ such that $\sigma(x, y)=1$ in a neighborhood of the diagonal $\{(x, x): x \in \bar{\Omega}\}$ in $\bar{\Omega} \times \bar{\Omega}$. The function $\sigma(x, y)$ depends on the shape of the domain $\Omega$; more precisely, it depends on a family of local charts on $\bar{\Omega}$. For example, if $\Omega$ is convex, one may take $\sigma(x, y) \equiv 1$ on $\bar{\Omega} \times \bar{\Omega}$.

(6) $W 1(x)=c(x)+\int_{\Omega} s(x, y)[1-\sigma(x, y)] d y \leq 0$ in $\Omega$, and $W 1 \not \equiv 0$ in $\Omega$.

The operator $W$ is called a second-order Waldenfels operator (cf. $[\mathbf{2}, \mathbf{8}]$ ). The differential operator $P$ is called a diffusion operator which describes analytically a strong Markov process with continuous paths (diffusion process) in the interior $\Omega$. The integro-differential operator $S_{r}$ is called a second-order Lévy operator which is supposed to correspond to the jump phenomenon in the interior $\Omega$. Therefore, the Waldenfels operator $W$ is supposed to correspond to such a diffusion phenomenon that a Markovian particle moves both by jumps and continuously in the state space $\Omega$.

We remark that the integro-differential operator $S_{r}$ is a "regularization" of $S$, since the integrand is absolutely convergent. Indeed, it suffices to note (see [5, Chapitre IV, Proposition 1]) that, for any compact $K \subset \mathbf{R}^{n}$, there exists a constant $C_{K}>0$ such that the distribution kernel $s(x, y)$ satisfies the estimate

$$
|s(x, y)| \leq \frac{C_{K}}{|x-y|^{n+2-\kappa}}, \quad x, y \in K, x \neq y
$$

The intuitive meaning of condition (6) is that the jump phenomenon from a point $x \in \Omega$ to the outside of a neighborhood of $x$ in $\Omega$ is "dominated" by the absorption phenomenon at $x$. In particular, if $c(x) \equiv 0$ in $\Omega$, then condition (6) implies that any Markovian particle does not move by jumps from $x \in \Omega$ to the outside of a neighborhood $V(x)$ of $x$ in the interior $\Omega$, since we have

$$
\int_{\Omega} s(x, y)[1-\sigma(x, y)] d y=0
$$

and so by conditions (4) and (5)

$$
s(x, y)=0, \quad y \in \Omega \backslash V(x) .
$$

Let $L$ be a first-order, boundary condition with real coefficients such that

$$
L u\left(x^{\prime}\right)=\mu\left(x^{\prime}\right) \frac{\partial u}{\partial \boldsymbol{\nu}}\left(x^{\prime}\right)+\gamma\left(x^{\prime}\right) u\left(x^{\prime}\right)+\int_{\Omega} r\left(x^{\prime}, y\right)\left[u(y)-u\left(x^{\prime}\right)\right] d y
$$

Here:

(1) $\mu \in C^{\infty}(\partial \Omega)$ and $\mu \geq 0$ on $\partial \Omega$. 
(2) $\gamma \in C^{\infty}(\partial \Omega)$ and $\gamma \leq 0$ on $\partial \Omega$.

(3) $\boldsymbol{\nu}=\left(\nu_{1}, \nu_{2}, \cdots, \nu_{n}\right)$ is the unit interior normal to the boundary $\partial \Omega$.

(4) The integral kernel $r(x, y)$ is the distribution kernel of a properly supported, pseudo-differential operator $R \in L_{1,0}^{-\theta}\left(\mathbf{R}^{n}\right), \theta>0$, which has the transmission property with respect to the boundary $\partial \Omega$, and $r(x, y) \geq 0$ off the diagonal in $\mathbf{R}^{n} \times \mathbf{R}^{n}$.

The boundary condition $L$ is called a first-order Ventcel' boundary condition (cf. $[\mathbf{2}, \mathbf{8}])$. The three terms of $L$

$$
\mu\left(x^{\prime}\right) \frac{\partial u}{\partial \boldsymbol{\nu}}\left(x^{\prime}\right), \quad \gamma\left(x^{\prime}\right) u\left(x^{\prime}\right), \quad \int_{\Omega} r\left(x^{\prime}, y\right)\left[u(y)-u\left(x^{\prime}\right)\right] d y
$$

are supposed to correspond to the reflection phenomenon, the absorption phenomenon and the inward jump phenomenon from the boundary, respectively.

In this paper, we prove an existence and uniqueness theorem for the following boundary value problem in the framework of Sobolev spaces of $L^{p}$ style:

$$
\begin{cases}W u=f & \text { in } \Omega, \\ L u=\varphi & \text { on } \partial \Omega .\end{cases}
$$

We remark that the note $[\mathbf{1 0}]$ only treated the case when $S=0$ and $R=0$, while the paper [9] studied problem $(*)$ in the framework of Sobolev spaces of $L^{2}$ style.

The function spaces we shall treat are the following (cf. [1, 12]): If $1<p<\infty$ and $m$ is a nonnegative integer, we define the Sobolev space

$$
\begin{aligned}
H^{m, p}(\Omega)= & \text { the space of functions } u \in L^{p}(\Omega) \text { whose } \\
& \text { derivatives } D^{\alpha} u,|\alpha| \leq m, \text { in the sense of } \\
& \text { distributions are in } L^{p}(\Omega) .
\end{aligned}
$$

The space $H^{m, p}(\Omega)$ is a Banach space with the norm

$$
\|u\|_{m, p}=\left(\sum_{|\alpha| \leq m} \int_{\Omega}\left|D^{\alpha} u(x)\right|^{p} d x\right)^{1 / p} .
$$

Furthermore, we let

$$
\begin{aligned}
B^{m-1 / p, p}(\partial \Omega)= & \text { the space of the boundary values } \varphi \text { of functions } \\
& u \in H^{m, p}(\Omega),
\end{aligned}
$$

and define a norm

$$
|\varphi|_{m-1 / p, p}=\inf \left\{\|u\|_{m, p}: u \in H^{m, p}(\Omega),\left.u\right|_{\partial \Omega}=\varphi\right\}
$$

The space $B^{m-1 / p, p}(\partial \Omega)$ is a Banach space with respect to the norm $|\cdot|_{m-1 / p, p}$; more precisely, it is a Besov space.

Our fundamental hypothesis for the boundary condition $L$ is the following:

$$
\gamma\left(x^{\prime}\right)-\int_{\Omega} r\left(x^{\prime}, y\right) d y<0 \quad \text { on the set } M=\left\{x^{\prime} \in \partial \Omega: \mu\left(x^{\prime}\right)=0\right\} .
$$


Intuitively, hypothesis (H) implies that, at any point of the set $M$ where no reflection phenomenon occurs, a Markovian particle may "disappear" or "move by jumps" to a random point in the interior $\Omega$.

We introduce a subspace of $B^{m-1-1 / p, p}(\partial \Omega)$ which is associated with the boundary condition $L$ in the following way: If we let

$$
\bar{\gamma}\left(x^{\prime}\right)=\gamma\left(x^{\prime}\right)-\int_{\Omega} r\left(x^{\prime}, y\right) d y
$$

then we find that condition $(\mathrm{H})$ is equivalent to the following one:

$$
\mu\left(x^{\prime}\right)-\bar{\gamma}\left(x^{\prime}\right)>0 \quad \text { on } \partial \Omega \text {. }
$$

We define the space

$$
\begin{aligned}
& B_{*}^{m-1-1 / p, p}(\partial \Omega)=\left\{\varphi=\mu \varphi_{1}-\bar{\gamma} \varphi_{2}: \varphi_{1} \in B^{m-1-1 / p, p}(\partial \Omega),\right. \\
&\left.\varphi_{2} \in B^{m-1 / p, p}(\partial \Omega)\right\},
\end{aligned}
$$

and a norm

$$
|\varphi|_{m-1-1 / p, p}^{*}=\inf \left\{\left|\varphi_{1}\right|_{m-1-1 / p, p}+\left|\varphi_{2}\right|_{m-1 / p, p}: \varphi=\mu \varphi_{1}-\bar{\gamma} \varphi_{2}\right\} .
$$

Then it is easy to verify that the space $B_{*}^{m-1-1 / p, p}(\partial \Omega)$ is a Banach space with respect to the norm $|\cdot|_{m-1-1 / p, p}^{*}$. We remark that the space $B_{*}^{m-1-1 / p, p}(\partial \Omega)$ is an "interpolation space" between the spaces $B^{m-1 / p, p}(\partial \Omega)$ and $B^{m-1-1 / p, p}(\partial \Omega)$. In particular, we have

$$
\begin{aligned}
& B_{*}^{m-1-1 / p, p}(\partial \Omega)=B^{m-1 / p, p}(\partial \Omega) \text { if } \mu \equiv 0 \text { on } \partial \Omega, \\
& B_{*}^{m-1-1 / p, p}(\partial \Omega)=B^{m-1-1 / p, p}(\partial \Omega) \text { if } \mu>0 \text { on } \partial \Omega .
\end{aligned}
$$

Now we can state our main result which is an $L^{p}$ version of $[\mathbf{9}$, Theorem 1] and is also a generalization of $[\mathbf{1 0}$, Theorem 1] to the case when $S \neq 0$ and $R \neq 0$.

Theorem. Let $1<p<\infty$ and let $m$ be a positive integer such that $m>1+1 / p$. If condition $(H)$ is satisfied, then the mapping

$$
(W, L): H^{m, p}(\Omega) \longrightarrow H^{m-2, p}(\Omega) \oplus B_{*}^{m-1-1 / p, p}(\partial \Omega)
$$

is an algebraic and topological isomorphism. In particular, for any $f \in H^{m-2, p}(\Omega)$ and any $\varphi \in B_{*}^{m-1-1 / p, p}(\partial \Omega)$, there exists a unique solution $u \in H^{m, p}(\Omega)$ of problem $(*)$.

Our approach has a great advantage of intuitive interpretation of the sufficient condition $(\mathrm{H})$ for the unique solvability of problem $(*)$ in terms of Markovian motion. In fact, Theorem states that if a Markovian particle moves incessantly both by jumps and continuously in the state space $\bar{\Omega}=\Omega \cup \partial \Omega$, not being trapped in the set $M$ where no reflection phenomenon occurs, then problem $(*)$ is uniquely solvable in the framework of Sobolev spaces of $L^{p}$ style.

Theorem will play an important role in the study of initial boundary value problems for semilinear parabolic differential equations as in [10].

I am grateful to the referee for his careful reading of the first draft of the manuscript and many valuable suggestions. 


\section{Proof of Theorem}

We begin by showing that the mapping

$$
(W, L): H^{m, p}(\Omega) \longrightarrow H^{m-2, p}(\Omega) \oplus B_{*}^{m-1-1 / p, p}(\partial \Omega)
$$

is continuous. To do this, we have only to verify the continuity of $L$.

By condition $(\mathrm{H})$, one can write the boundary condition $L u$ in the following form:

$$
\begin{aligned}
L u & =\mu \frac{\partial u}{\partial \boldsymbol{\nu}}+\gamma u+\int_{\Omega} r(\cdot, y)[u(y)-u(\cdot)] d y \\
& =\mu \frac{\partial u}{\partial \boldsymbol{\nu}}+\bar{\gamma} u+R\left(u^{0}\right) \\
& =\mu\left(\frac{\partial u}{\partial \boldsymbol{\nu}}+\frac{1}{\mu-\bar{\gamma}} R\left(u^{0}\right)\right)-\bar{\gamma}\left(\frac{1}{\mu-\bar{\gamma}} R\left(u^{0}\right)-u\right) \quad \text { on } \partial \Omega
\end{aligned}
$$

where

$$
u^{0}(x)= \begin{cases}u(x) & \text { if } x \in \bar{\Omega}, \\ 0 & \text { if } x \in \mathbf{R}^{n} \backslash \bar{\Omega} .\end{cases}
$$

Since $R \in L_{1,0}^{-\theta}\left(\mathbf{R}^{n}\right)$ has the transmission property, it follows (see [7, Section 2.3]) that the operator $R_{\Omega}$, defined by the formula

$$
R_{\Omega} u=\left.R\left(u^{0}\right)\right|_{\Omega}
$$

maps $H^{s, p}(\Omega)$ continuously into $H^{s+\theta, p}(\Omega)$ for all $s \geq 0$. Hence we find from the definition of $B_{*}^{m-1-1 / p, p}(\partial \Omega)$ that the operator $L: H^{m, p}(\Omega) \rightarrow B_{*}^{m-1-1 / p, p}(\partial \Omega)$ is continuous.

Therefore, in order to prove Theorem, it suffices to show that the mapping $(W, L)$ is bijective. Indeed, the continuity of the inverse of $(W, L)$ follows from an application of Banach's closed graph theorem, since $(W, L)$ is continuous.

The proof is divided into five steps.

(I) First we show that the boundary value problem

$$
\begin{cases}W u=f & \text { in } \Omega, \\ L u=\varphi & \text { on } \partial \Omega\end{cases}
$$

can be reduced to the study of an operator on the boundary.

Now let $f$ be an arbitrary element of $H^{m-2, p}(\Omega)$ and let $\varphi$ be an arbitrary element of $B_{*}^{m-1-1 / p, p}(\partial \Omega)$ such that

$$
\varphi=\mu \varphi_{1}-\bar{\gamma} \varphi_{2}, \quad \varphi_{1} \in B^{m-1-1 / p, p}(\partial \Omega), \varphi_{2} \in B^{m-1 / p, p}(\partial \Omega) .
$$

We consider the Neumann problem

$$
\begin{cases}W v=f & \text { in } \Omega, \\ \frac{\partial v}{\partial \boldsymbol{\nu}}=\varphi_{1} & \text { on } \partial \Omega .\end{cases}
$$

The existence and uniqueness theorem for problem $(\mathrm{N})$ is well established in the framework of Sobolev spaces of $L^{p}$ style (see [11, Chapter XI]). Indeed, it suffices 
to note that the operator $W=P+S_{r}$ is a relatively compact perturbation of $P$ in the framework of Sobolev spaces of $L^{p}$ style, since $S_{r}$ is of order $2-\kappa<2$. We let

$$
v=\mathcal{G}_{N}\left(f, \varphi_{1}\right) \in H^{m, p}(\Omega) .
$$

The operator $\mathcal{G}_{N}$ is a generalization of the classical Green operator. Then it follows that a function $u$ is a solution of problem (*) if and only if the function $w=u-v$ is a solution of the problem

$$
\begin{cases}W w=0 & \text { in } \Omega \\ L w=\varphi-L v & \text { on } \partial \Omega .\end{cases}
$$

Here we remark that

$$
L v=\mu \frac{\partial v}{\partial \boldsymbol{\nu}}+\bar{\gamma} v+R_{\Omega} v=\mu \varphi_{1}+\bar{\gamma} v+R_{\Omega} v \quad \text { on } \partial \Omega,
$$

so that

$$
L w=\varphi-L v=-\bar{\gamma}\left(\varphi_{2}+v\right)-R_{\Omega} v \in B^{m-1 / p, p}(\partial \Omega) .
$$

But we know that every solution $w \in H^{m, p}(\Omega)$ of the homogeneous equation $W w=$ 0 can be expressed as follows.

$$
w=\mathcal{P} \psi, \quad \psi \in B^{m-1 / p, p}(\partial \Omega) .
$$

The operator $\mathcal{P}$ is a generalization of the classical Poisson operator; that is, the function $w=\mathcal{P} \psi$ is the unique solution of the Dirichlet problem

$$
\begin{cases}W w=0 & \text { in } \Omega, \\ w=\psi & \text { on } \partial \Omega .\end{cases}
$$

Thus, by using the operators $\mathcal{G}_{N}$ and $\mathcal{P}$, one can reduce the study of problem $(*)$ to that of the equation

$$
T \psi:=L \mathcal{P} \psi=-\bar{\gamma}\left(\varphi_{2}+v\right)-R_{\Omega} v \quad \text { on } \partial \Omega .
$$

This is a generalization of the classical Fredholm integral equation.

More precisely, we can formulate this reduction to the boundary in the framework of Sobolev spaces of $L^{p}$ style as follows:

Proposition 2.1. Let $1<p<\infty$ and $m>1+1 / p$. For given functions $f \in$ $H^{m-2, p}(\Omega)$ and $\varphi=\mu \varphi_{1}-\bar{\gamma} \varphi_{2} \in B_{*}^{m-1-1 / m, p}(\partial \Omega)$, there exists a solution $u \in$ $H^{m, p}(\Omega)$ of problem $(*)$ if and only if there exists a solution $\psi \in B^{m-1 / p, p}(\partial \Omega)$ of equation $(\dagger)$.

(II) Next we show that the operator

$$
\begin{aligned}
T \psi\left(x^{\prime}\right) & =L \mathcal{P} \psi\left(x^{\prime}\right) \\
& =\mu\left(x^{\prime}\right) \frac{\partial}{\partial \boldsymbol{\nu}}(\mathcal{P} \psi)\left(x^{\prime}\right)+\gamma\left(x^{\prime}\right) \psi\left(x^{\prime}\right)+\int_{\Omega} r\left(x^{\prime}, y\right)\left[\mathcal{P} \psi(y)-\psi\left(x^{\prime}\right)\right] d y
\end{aligned}
$$

is a first-order, pseudo-differential operator on the boundary $\partial \Omega$. 
In fact, we have the following results:

(a) It is known (see [6, Chapter XX], [7, Chapter 3]) that the operator

$$
\Pi: \psi\left(x^{\prime}\right) \longmapsto \frac{\partial}{\partial \boldsymbol{\nu}}(\mathcal{P} \psi)\left(x^{\prime}\right)
$$

is a first-order, elliptic pseudo-differential operator on $\partial \Omega$. For example, if $W$ is the usual Laplacian $\Delta=\partial^{2} / \partial x_{1}^{2}+\partial^{2} / \partial x_{2}^{2}+\cdots+\partial^{2} / \partial x_{n}^{2}$, that is, if $P=\Delta$ and $S=0$, then it follows that the principal symbol $p_{1}\left(x^{\prime}, \xi^{\prime}\right)$ of $\Pi$ is given by the formula

$$
\begin{aligned}
p_{1}\left(x^{\prime}, \xi^{\prime}\right)= & \text { minus the length }\left|\xi^{\prime}\right| \text { of } \xi^{\prime} \text { with respect to the Riemannian } \\
& \text { metric of } \partial \Omega \text { induced by the natural metric of } \mathbf{R}^{n} .
\end{aligned}
$$

(b) Since the pseudo-differential operator $R \in L_{1,0}^{-\theta}\left(\mathbf{R}^{n}\right)$ has the transmission property with respect to $\partial \Omega$, it follows (see $[\mathbf{4}],[\mathbf{7}$, Chapter 2]) that the operator

$$
Q: \psi\left(x^{\prime}\right) \longmapsto \int_{\Omega} r\left(x^{\prime}, y\right) \mathcal{P} \psi(y) d y
$$

is a pseudo-differential operator of order $-\theta$ on $\partial \Omega$.

(III) We study the pseudo-differential operator $T$ in question. The next proposition is an essential step in the proof of Theorem.

Proposition 2.2. If hypothesis $(H)$ is satisfied, then there exists a parametrix $E$ in the Hörmander class $L_{1,1 / 2}^{0}(\partial \Omega)$ for $T$ such that

$$
\begin{aligned}
& E T \equiv T E \equiv I \quad \bmod L^{-\infty}(\partial \Omega), \\
& E: B^{\sigma, p}(\partial \Omega) \longrightarrow B^{\sigma, p}(\partial \Omega) \quad \text { for all } \sigma \in \mathbf{R} .
\end{aligned}
$$

Proof. By assertions (a) and (b), it follows that the pseudo-differential operator $T$ can be written in the form

$$
T=\mu \Pi+\bar{\gamma}+Q
$$

where $\Pi$ is of order 1 and $Q$ is of order $-\theta$, respectively. If the symbol of $\Pi$ has an asymptotic expansion

$$
p_{1}\left(x^{\prime}, \xi^{\prime}\right)+p_{0}\left(x^{\prime}, \xi^{\prime}\right)+\cdots,
$$

then we find that the symbol $t\left(x^{\prime}, \xi^{\prime}\right)$ of $T$ is given by the formula

$$
\begin{aligned}
t\left(x^{\prime}, \xi^{\prime}\right)= & \mu\left(x^{\prime}\right)\left(\operatorname{Re} p_{1}\left(x^{\prime}, \xi^{\prime}\right)+\sqrt{-1} \operatorname{Im} p_{1}\left(x^{\prime}, \xi^{\prime}\right)\right) \\
& +\left(\left[\bar{\gamma}\left(x^{\prime}\right)+\mu\left(x^{\prime}\right) \operatorname{Re} p_{0}\left(x^{\prime}, \xi\right)\right]+\sqrt{-1} \mu\left(x^{\prime}\right) \operatorname{Im} p_{0}\left(x^{\prime}, \xi\right)\right) \\
& + \text { terms of negative order. }
\end{aligned}
$$

Here we remark that:

(1) Re $p_{1}\left(x^{\prime}, \xi^{\prime}\right)<0$ on the bundle $T^{*}(\partial \Omega) \backslash\{0\}$ of non-zero cotangent vectors.

(2) Hypothesis $(\mathrm{H})$ implies that

$$
\bar{\gamma}\left(x^{\prime}\right)<0 \quad \text { on the set } M=\left\{x^{\prime} \in \partial \Omega: \mu\left(x^{\prime}\right)=0\right\} \text {. }
$$

Thus, just as in [10, Lemma 5.3], one can prove the following: 
Claim. Assume that hypothesis $(H)$ is satisfied. Then, for each point $x_{0}^{\prime}$ of $\partial \Omega$, there exists a neighborhood $U\left(x_{0}^{\prime}\right)$ of $x_{0}^{\prime}$ such that:

For any compact $K \subset U\left(x_{0}^{\prime}\right)$ and any multi-indices $\alpha, \beta$, there exist constants $C_{K, \alpha, \beta}>0$ and $C_{K}>0$ such that we have, for all $x^{\prime} \in K$ and $\left|\xi^{\prime}\right| \geq C_{K}$,

$$
\begin{aligned}
\left|D_{\xi^{\prime}}^{\alpha} D_{x^{\prime}}^{\beta} t\left(x^{\prime}, \xi^{\prime}\right)\right| \leq & C_{K, \alpha, \beta}\left|t\left(x^{\prime}, \xi^{\prime}\right)\right|\left(1+\left|\xi^{\prime}\right|\right)^{-|\alpha|+(1 / 2)|\beta|}, \\
& \left|t\left(x^{\prime}, \xi^{\prime}\right)\right|^{-1} \leq C_{K} .
\end{aligned}
$$

Therefore, applying [6, Theorem 22.1.3] to our situation, one can construct a parametrix $E \in L_{1,1 / 2}^{0}(\partial \Omega)$ for $T$.

The boundedness of the parametrix $E: B^{\sigma, p}(\partial \Omega) \rightarrow B^{\sigma, p}(\partial \Omega)$ follows from an application of a Besov-space boundedness theorem due to Bourdaud [3, Theorem 1].

(IV) We associate with equation $(\dagger)$ a linear operator

$$
\mathcal{T}: B^{m-1 / p, p}(\partial \Omega) \longrightarrow B^{m-1 / p, p}(\partial \Omega)
$$

as follows.

$(\alpha)$ The domain $D(\mathcal{T})$ of $\mathcal{T}$ is the space

$$
D(\mathcal{T})=\left\{\psi \in B^{m-1 / p, p}(\partial \Omega): T \psi \in B^{m-1 / p, p}(\partial \Omega)\right\} .
$$

( $\beta) \mathcal{T} \psi=T \psi, \psi \in D(\mathcal{T})$.

Then the operator $\mathcal{T}$ is a densely defined, closed linear operator, since the operator $T: B^{m-1 / p, p}(\partial \Omega) \rightarrow B^{m-1-1 / p, p}(\partial \Omega)$ is continuous and since the domain $D(\mathcal{T})$ contains the space $C^{\infty}(\partial \Omega)$.

Furthermore, just as in the proof of [10, Theorem 6.6], we can obtain the following results for the operator $\mathcal{T}$ :

Theorem 2.3. Assume that hypothesis $(H)$ is satisfied. Then we have the following:

(i) The operator $\mathcal{T}$ is a Fredholm operator with index zero, that is,

$$
\text { ind } \mathcal{T}:=\operatorname{dim} N(\mathcal{T})-\operatorname{codim} R(\mathcal{T})=0 \text {. }
$$

(ii) The null space

$$
N(\mathcal{T})=\left\{\psi \in B^{m-1 / p, p}(\partial \Omega): T \psi=0\right\}
$$

consists of $C^{\infty}$ functions on $\partial \Omega$.

Proof. In order to prove assertion (i), we write the boundary condition $L u$ as

$$
\begin{aligned}
L u & =\left(\mu \frac{\partial u}{\partial \boldsymbol{\nu}}+\bar{\gamma} u\right)+\int_{\Omega} r(\cdot, y) u(y) d y \\
& :=L_{0} u+R_{\Omega} u .
\end{aligned}
$$


Then it follows that the operator

$$
(W, L)=\left(P+S_{r}, L_{0}+R_{\Omega}\right): H^{m, p}(\Omega) \longrightarrow H^{m-2, p}(\Omega) \times B_{*}^{m-1-1 / p, p}(\partial \Omega)
$$

is a relatively compact perturbation of the operator

$$
\left(P, L_{0}\right): H^{m, p}(\Omega) \longrightarrow H^{m-2, p}(\Omega) \times B_{*}^{m-1-1 / p, p}(\partial \Omega),
$$

since $S_{r}$ is of order $2-\kappa<2$ and $R_{\Omega}$ is of order $-\theta<0$. But, arguing as in the proof of $[\mathbf{1 0}$, Theorem 6.6], we find that if hypothesis $(\mathrm{H})$ is satisfied, then we have

$$
\text { ind }\left(P, L_{0}\right)=0 \text {. }
$$

Therefore, by using Proposition 2.1, we obtain that

$$
\text { ind } \mathcal{T}=\operatorname{ind}(W, L)=\text { ind }\left(P, L_{0}\right)=0 .
$$

Assertion (ii) is an immediate consequence of Proposition 2.2.

(V) Finally we prove that the mapping $(W, L)$ is bijective, or equivalently,

$$
\left\{\begin{array}{l}
\text { Problem }(*) \text { has a unique solution } u \in H^{m, p}(\Omega) \text { for } \\
\text { any } f \in H^{m-2, p}(\Omega) \text { and any } \varphi \in B_{*}^{m-1-1 / p, p}(\partial \Omega) .
\end{array}\right.
$$

$(\mathrm{V}-1)$ In doing so, we first prove uniqueness result for problem $(*)$ :

$$
\left\{\begin{array}{l}
u \in H^{m, p}(\Omega), W u=0 \text { in } \Omega, L u=0 \text { on } \partial \Omega \\
\Longrightarrow u=0 \text { in } \Omega .
\end{array}\right.
$$

By virtue of Proposition 2.1, we find from part (ii) of Theorem 2.3 that

$$
\left\{\begin{array}{l}
u \in H^{m, p}(\Omega), W u=0 \text { in } \Omega, L u=0 \text { on } \partial \Omega \\
\Longrightarrow u \in C^{\infty}(\bar{\Omega}) .
\end{array}\right.
$$

Therefore, uniqueness result (2.2) is an immediate consequence of the following maximum principle:

Proposition 2.4. If hypothesis (H) is satisfied, then we have

$$
\left\{\begin{array}{l}
u \in C^{2}(\bar{\Omega}), W u \geq 0 \text { in } \Omega, L u \geq 0 \text { on } \partial \Omega \\
\Longrightarrow u \leq 0 \text { on } \bar{\Omega} .
\end{array}\right.
$$

Proof. If $u$ is a constant $C$, then it follows that

$$
0 \leq W u=C W 1 \quad \text { in } \Omega
$$

This implies that $C$ is nonpositive, since $W 1 \leq 0$ and $W 1 \not \equiv 0$ in $\Omega$.

Hence we have only to consider the case when $u$ is not a constant. Assume to the contrary that

$$
C=\max _{\bar{\Omega}} u>0
$$


Then, applying the strong maximum principle (see Appendix, Theorem A.1) to the operator $W$, we obtain that there exists a point $x_{0}^{\prime}$ of $\partial \Omega$ such that

$$
\left\{\begin{array}{l}
u\left(x_{0}^{\prime}\right)=C>0 \\
u(y)<u\left(x_{0}^{\prime}\right) \text { for all } y \in \Omega .
\end{array}\right.
$$

Furthermore, it follows from an application of the boundary point lemma (see Theorem A.2) that

$$
\frac{\partial u}{\partial \nu}\left(x_{0}^{\prime}\right)<0 .
$$

This implies that $\mu\left(x_{0}^{\prime}\right)=0$, that is,

$$
x_{0}^{\prime} \in M=\left\{x^{\prime} \in \partial \Omega: \mu\left(x^{\prime}\right)=0\right\},
$$

since we have

$$
0 \leq L u\left(x_{0}^{\prime}\right) \leq \mu\left(x_{0}^{\prime}\right) \frac{\partial u}{\partial \nu}\left(x_{0}^{\prime}\right) .
$$

Therefore, by hypothesis $(\mathrm{H})$ and assertion (2.3), we find that

$$
L u\left(x_{0}^{\prime}\right)=\gamma\left(x_{0}^{\prime}\right) u\left(x_{0}^{\prime}\right)+\int_{\Omega} r\left(x_{0}^{\prime}, y\right)\left[u(y)-u\left(x_{0}^{\prime}\right)\right] d y<0 .
$$

This contradicts the hypothesis: $L u \geq 0$ on $\partial \Omega$.

(V-2) In view of Proposition 2.1, we obtain from uniqueness result (2.2) that the operator $\mathcal{T}: B^{m-1 / p, p}(\partial \Omega) \rightarrow B^{m-1 / p, p}(\partial \Omega)$ is injective, that is,

$$
\operatorname{dim} N(\mathcal{T})=0 .
$$

But, part (i) of Theorem 2.3 tells us that $\mathcal{T}$ is a Fredholm operator with index zero. Thus we have

$$
\operatorname{codim} R(\mathcal{T})=0,
$$

which proves that $\mathcal{T}$ is surjective. Hence it follows that the operator $\mathcal{T}$ is bijective. Therefore, existence and uniqueness result (2.1) follows by using again Proposition 2.1 .

The proof of Theorem is now complete.

\section{Appendix: The maximum principle}

In this appendix, following Bony-Courrège-Priouret [2], we formulate two useful maximum principles for second-order elliptic Waldenfels operators.

First we state the strong maximum principle (see [2, Théorème VII]):

Theorem A.1. Let $W$ be a second-order elliptic Waldenfels operator. Assume that

$$
\left\{\begin{array}{l}
u \in C^{2}(\bar{\Omega}), W u \geq 0 \quad \text { in } \Omega, \\
C=\max _{\bar{\Omega}} u \geq 0 .
\end{array}\right.
$$

If the function $u$ takes its maximum $C$ at an interior point $x_{0} \in \Omega$, then $u \equiv C$ in the connected component containing $x_{0}$.

Next we consider the interior normal derivative $(\partial u) /(\partial \boldsymbol{\nu})$ at a boundary point where the function $u \in C^{2}(\bar{\Omega})$ takes its nonnegative maximum.

The boundary point lemma reads as follows (see [2, Théorème VIII]): 
Theorem A.2. Let $W$ be a second-order elliptic Waldenfels operator. Assume that

$$
\left\{\begin{array}{l}
u \in C^{2}(\bar{\Omega}), W u \geq 0 \quad \text { in } \Omega, \\
C=\max _{\bar{\Omega}} u \geq 0 .
\end{array}\right.
$$

If the function $u$ takes its maximum $C$ at a boundary point $x_{0}^{\prime} \in \partial \Omega$, then either $u \equiv C$ in the connected component containing $x_{0}^{\prime}$ or

$$
\frac{\partial u}{\partial \boldsymbol{\nu}}\left(x_{0}^{\prime}\right)<0
$$

\section{References}

1. J. Bergh and J. Löfström, Interpolation spaces, an introduction, Springer-Verlag, Berlin, 1976.

2. J.-M. Bony, P. Courrège et P. Priouret, Semi-groupes de Feller sur une variété à bord compacte et problèmes aux limites intégro-différentiels du second ordre donnant lieu au principe du maximum, Ann. Inst. Fourier (Grenoble) 18 (1968), 369-521.

3. G. Bourdaud, $L^{p}$-estimates for certain non-regular pseudo-differential operators, Comm. Partial Differential Equations 7 (1982), 1023-1033.

4. L. Boutet de Monvel, Boundary problems for pseudo-differential operators, Acta Math. 126 (1971), 11-51.

5. R. R. Coifman et Y. Meyer, Au-delà des opérateurs pseudo-différentiels, Astérisque No. 57, Soc. Math. France, Paris, 1978.

6. L. Hörmander, The analysis of linear partial differential operators III, Springer-Verlag, Berlin Heidelberg New York Tokyo, 1985.

7. S. Rempel and B.-W. Schulze, Index theory of elliptic boundary problems, Akademie-Verlag, Berlin, 1982.

8. K. Taira, On the existence of Feller semigroups with boundary conditions, Memoirs Amer. Math. Soc. No. 475 (1992).

9. K. Taira, Boundary value problems for elliptic pseudo-differential operators, Proc. Amer. Math. Soc. 123 (1995), 2519-2528.

10. K. Taira, Analytic semigroups and semilinear initial boundary value problems, London Mathematical Society Lecture Note Series, No. 223, Cambridge University Press, London New York, 1995.

11. M. Taylor, Pseudodifferential operators, Princeton Univ. Press, Princeton, 1981.

12. H. Triebel, Interpolation theory, function spaces, differential operators, North-Holland, Amsterdam, 1978 . 